\title{
Reinserção social de dependentes químicos residentes em comunidades terapêuticas ${ }^{1}$
}

\author{
Kévin da Silva Souza² \\ Iuri Fernando Coutinho e Silva ${ }^{2}$ \\ Sonis Henrique Rezende Batista ${ }^{3}$ \\ Rogério José de Almeida ${ }^{4}$
}

O estudo teve por objetivo caracterizar as ações e atividades voltadas para a reinserção social de dependentes químicos residentes em comunidades terapêuticas. Foram avaliadas 43 comunidades, localizadas no estado de Goiás, com a utilização de um questionário semiestruturado. Os dados foram analisados por meio de estatística descritiva e os resultados apontaram que essas comunidades apresentam mecanismos de preparo do residente para a alta terapêutica e encaminhamento ao mercado de trabalho. Entretanto, desenvolvem poucas atividades para a inserção no mercado de trabalho. O envolvimento da família no tratamento dos residentes é estimulado com visitas e atividades conjuntas. Conclui-se que embora o trabalho das comunidades seja relevante, ainda carece de ajuda de políticas públicas de assistência social que contribuam com a reinserção desses indivíduos na sociedade.

Descritores: Dependência Química; Drogas; Tratamento; Reinserção Social.

\footnotetext{
${ }^{1}$ Apoio Financeiro da Fundação de Amparo à Pesquisa do Estado de Goiás (FAPEG), processo n 201210267001235. ${ }^{2}$ Aluno do curso de graduação em Medicina, Pontifícia Universidade Católica de Goiás, Goiás, Goiânia, GO, Brasil.

${ }^{3} \mathrm{MSc}$.

${ }^{4}$ Pós-doutorando, Professor Ajunto, Pontifícia Universidade Católica de Goiás, Faculdade de Medicina, Goiânia, GO, Brasil.
} 


\title{
Social reintegration of drug-addicted individuals living in therapeutic communities
}

The study aimed to characterize actions and activities aimed at social reintegration of drugaddicted living in therapeutic communities. Forty-three communities were evaluated in the state of Goiás, with the use of a semi-structured questionnaire. Data were analyzed using descriptive statistics and the results showed that these communities have resident preparation mechanisms for therapeutic discharge and referral to the labor market. However, few activities are developed for inclusion in the labor market. Family's involvement in treatment of residents is stimulated with joint visits and activities. Although the relevant work of communities, help from public policies is needed for social assistance to contribute to the reintegration of these individuals in society.

Descriptors: Dependence; Drugs; Treatment; Social Reintegration.

\section{Reinserción social de dependientes químicos residentes en comunidades terapéuticas}

\begin{abstract}
El estudio tuvo por objetivo caracterizar las acciones y actividades vueltas para la reinserción social de dependientes químicos residentes en comunidades terapéuticas. Fueron evaluadas 43 comunidades, localizadas en el estado de Goiás, con la utilización de un cuestionario semiestructurado. Los datos fueron analizados por medio de estadística descriptiva y los resultados apuntaron que esas comunidades presentan mecanismos de preparo del residente para la alta terapéutica y encaminamiento al mercado de trabajo. Mientras, desarrollan pocas actividades para la inserción en el mercado de trabajo. El envolvimiento de la familia en el tratamiento de los residentes es estimulado con visitas y actividades conjuntas. Se concluye que aunque el trabajo de las comunidades sea relevante, aún carece de ayuda de políticas públicas de asistencia social que aporten con la reinserción de eses individuos en la sociedad.
\end{abstract}

Descriptores: Abuso de Sustancias; Drogas; Tratamiento; Reinserción Social.

\section{Introdução}

O uso ou abuso de drogas lícitas e ilícitas tem aumentado substancialmente nos últimos anos. De acordo com o Relatório Mundial sobre Drogas de 2012, de $3,4 \%$ a $6,6 \%$ da população entre 15 e 64 anos é usuária (1). Este relatório apontou que em 2015 essa prevalência do uso se manteve estável ${ }^{(2)}$. A estimativa é que 1 em cada 10 usuários de drogas apresenta algum transtorno psiquiátrico ou dependência(2).

O uso dessas substâncias está concentrado na população jovem urbana, com ênfase para os países em desenvolvimento. Neste contexto, se destaca o Brasil, considerado o maior mercado consumidor de cocaína da América do Sul. Calcula-se que 1,75\% de sua população adulta seja usuária dessa droga ${ }^{(2)}$. 
Com o propósito de alterar esse panorama, o Governo Federal instituiu a "Rede de Atenção Psicossocial para pessoas com sofrimento ou transtorno mental e com necessidades decorrentes do uso de crack, álcool e outras drogas", por meio da Portaria n. 3088, de 23 de dezembro de 2011. Essa Portaria busca instituir a prevenção e redução de danos provocados pelo consumo de crack, álcool e outras drogas, assim como a promoção da reabilitação e reinserção das pessoas com transtorno mental e com necessidades decorrentes desse consumo na sociedade, por meio do acesso ao trabalho, renda e moradia solidária(3). Essa ação governamental também prevê a criação, ampliação e articulação de serviços de cuidado à saúde de pessoas com transtorno mental ou em situação de uso, abuso ou dependência de álcool, crack e outras drogas ${ }^{(3)}$.

Dentre esses serviços, destacam-se aqueles de atenção residencial de caráter transitório, categoria na qual se enquadram as comunidades terapêuticas. Essas comunidades têm como objetivo oferecer cuidados contínuos de saúde, por até nove meses para adultos que tenham necessidades clínicas estáveis( ${ }^{(3)}$. Atuam como instituições sociais que buscam reabilitar o dependente químico, através da promoção da mudança de comportamento(4). Além disso, devem, ainda, funcionar de forma articulada com a atenção básica e com o Centro de Atenção Psicossocial - CAPS $^{(3)}$.

Nessas instituições são realizadas intervenções como reabilitação física e psicológica, recuperação, resgate da cidadania e reinserção social do indivíduo dependente por meio de um programa terapêutico individualizado que pode envolver: laborterapia, convivência comunitária, desenvolvimento de atividades profissionalizantes, programas culturais, atividades religiosas e espirituais, entre outras ${ }^{(5)}$.

Dentre essas múltiplas atuações, merece relevância a reinserção social do indivíduo. Esta é uma ação complexa, que envolve o resgate da autoestima e a valorização das capacidades individuais em prol de toda a coletividade ${ }^{(6)}$.

Essa prática deve, portanto, envolver a superação da insegurança quanto ao retorno do usuário ao convívio familiar e à disponibilização de acesso a programas voltados para a geração de renda, profissionalização e participação comunitária, alcançando assim a recuperação integral do indivíduo ${ }^{(7)}$. Espera-se que os sujeitos desenvolvam habilidades para viver, aprender, aprimorar sua convivência em sociedade e ampliar sua autonomia( ${ }^{(8)}$.

Toda atividade que objetiva à reinserção social deve enfatizar as qualidades e os potenciais do indivíduo com a reconstrução de três aspectos principais: casa, trabalho e rede social. Trata-se de um fenômeno intrapessoal e individual, não seguindo um padrão entre os dependentes químicos ${ }^{(9)}$.

$\mathrm{Na}$ reinserção social, a família, como rede social primária do indivíduo e codependente desempenha um papel fundamental. A forma como o indivíduo é acolhido e como as relações se restabelecem entre ele e seus familiares são importantes para a sua segurança emocional e social, proporcionandoIhe condições favoráveis para manter-se em abstinência ${ }^{(10)}$. Entretanto, essa reinserção social nem sempre ocorre e faltam políticas públicas que apoiem essa iniciativa. O preconceito e a discriminação ainda dificultam a prática da cidadania. A população deve se conscientizar de que os indivíduos em abstinência também são cidadãos e, como tal, têm direitos e deveres perante a sociedade ${ }^{(10)}$.

Os próprios profissionais de saúde que trabalham em centros de referência de dependência química muitas vezes têm um conhecimento limitado sobre essa problemática. Fato esse demonstrado em um estudo que identificou que a reinserção social, no entendimento de muitos profissionais, se restringe à realização de oficinas terapêuticas, atividades externas e parceria com outras instituições, evidenciando uma visão atrelada ao modelo psiquiátrico tradicional(11). Portanto, faz-se necessário investigar o papel exercido atualmente pelas comunidades terapêuticas na reintegração social de dependentes químicos.

Assim, o objetivo do presente estudo é caracterizar as ações e atividades voltadas para a reinserção social de dependentes químicos residentes em comunidades terapêuticas, com ênfase no preparo para a alta terapêutica, o encaminhamento para o mercado de trabalho e a existência e funcionamento de parcerias entre essas comunidades e outras instituições.

\section{Materiais e Métodos}

Participaram da pesquisa 43 comunidades terapêuticas situadas no estado de Goiás, especificamente aquelas localizadas no município de Goiânia e região metropolitana, e da cidade de Anápolis, pela sua importância demográfica e proximidade com a capital. As comunidades participantes foram selecionadas por meio de um cadastro junto ao Fórum Goiano de Enfrentamento ao Crack e Outras Drogas e ao Grupo Executivo de Enfrentamento às Drogas - GEED. As visitas foram realizadas no período de agosto de 2014 a fevereiro de 2015. 
A região metropolitana de Goiânia é composta por 20 municípios, conforme dispõe a Lei Complementar Estadual $n^{\circ} 78$ de 25 de março de 2010(12). Destes, acrescido o de Anápolis, foram visitados 12, onde encontram-se comunidades terapêuticas que se enquadravam no modelo de acolhimento voluntário e manifestaram interesse em participar da pesquisa.

Através de contato telefônico procurouse identificar se a entidade era realmente um estabelecimento de acolhimento voluntário para dependentes químicos, explicando os objetivos da pesquisa, em breve relato dos procedimentos, comunicando a necessidade de promover uma visita e a coleta de informações com a aplicação de um questionário.

Optou-se pela utilização de um questionário semiestruturado aplicado pelo pesquisador para a obtenção dos dados gerais dos entrevistados e das instituições, serviços oferecidos pela comunidade, equipe de trabalho, público atendido e relação da comunidade com os serviços de saúde $^{(13)}$. As entrevistas foram gravadas, registrado fotograficamente a estrutura física, da mesma maneira que solicitados documentos sobre o funcionamento da entidade.

Precedendo a entrevista, o responsável pela instituição leu e assinou o Termo de Consentimento Livre e Esclarecido - TCLE. Esta pesquisa está inscrita na Comissão Nacional de Ética em Pesquisa - CONEP com a CAAE: 12416213.1.0000.0037 e foi aprovada pelo Comitê de Ética em Pesquisa - CEP da Pontifícia Universidade Católica de Goiás - PUC Goiás.

Após a aplicação do instrumento, foi elaborado um banco de dados utilizando o Programa estatístico SPSS versão 16. Realizou-se análise descritiva dos dados, apresentando-se a frequência absoluta, frequência relativa e medidas de tendência central (Média aritmética, mediana, soma, mínimo e máximo) e medidas de dispersão (Desvio padrão).

\section{Resultados}

Integraram a investigação 43 comunidades terapêuticas na área estudada, sendo todas de caráter não governamental. Um total de $27,9 \%$ dessas comunidades possuía uma mantenedora e dentre estas, $53,5 \%$ afirmaram ter uma orientação religiosa.

No que refere-se à propriedade, pouco mais da metade $(51,2 \%)$ afirmaram ser autônomas, $30,2 \%$ alugaram os estabelecimentos para o devido funcionamento e $18,6 \%$ funcionavam com sede cedida.

Em relação à definição do público-alvo, as comunidadesestudadasatendiampredominantemente usuários de drogas lícitas e ilícitas $(97,7 \%)$, sendo que $2,3 \%$ dessas instituições atendiam apenas usuários de drogas ilícitas. Os residentes eram em sua maioria, adultos do sexo masculino provenientes de zona urbana $(95,3 \%)$ e com renda mensal familiar média inferior a um salário mínimo (53,5\%).

No que se refere às atividades desenvolvidas nas comunidades terapêuticas que objetivavam à preparação do residente para a reinserção no mercado de trabalho, apenas $39,5 \%$ ofereciam oficinas profissionalizantes a seus residentes. Nesse percentual, as atividades aconteciam diariamente em $52,9 \%$ delas. De forma semanal em $35,3 \%$. Um curso de informática era oferecido também em pequena porcentagem $(23,3 \%)$, na sua maioria semanalmente.

Apoio educacional aos residentes era ofertado em $32,6 \%$ das comunidades terapêuticas. Os percentuais se equilibram tanto na forma diária quanto semanal: $42,9 \%$ e $50 \%$ respectivamente.

Um dos principais obstáculos enfrentados por essas comunidades, no que diz respeito à assistência aos seus tutelados, foi a reinserção no mundo do trabalho. Boa parte delas $(76,7 \%)$ tentava encaminhá-los ao término de sua estadia. Os resultados, entretanto, se apresentam pouco efetivos devido à precariedade e falta de planejamento estruturado. As evidências se fazem notar em sua grande maioria $(81,8 \%)$, sendo a grande parte desses encaminhamentos para empresas parceiras. Porém, até mesmo entre essas empresas, as comunidades terapêuticas relataram uma série de dificuldades, tais como: desconfiança, preconceito, falta de qualificação do residente e medo.

Pouco mais da metade $(51,2 \%)$ das instituições pesquisadas possuíam registro e acompanhamento do ex-residente em relação a sua reinserção social. Entretanto, em termos pragmáticos, essas afirmativas se diluíam, pois somente $18,6 \%$ afirmaram realizar e registrar um acompanhamento em relação à permanência do indivíduo no mercado de trabalho.

Com relação à importante participação da família na reabilitação integral do dependente químico, identificou-se que na quase totalidade das comunidades terapêuticas $(97,7 \%)$, as famílias realizavam visitas aos residentes, sendo que estas ocorriam predominantemente uma vez ao mês. Contudo, na maior parte das comunidades, havia regras estabelecidas e que precisavam ser atendidas. 
Em 81,4\% existiam critérios para a visita familiar, tais como: disciplina, seguimento das normas internas da comunidade, abstinência de drogas, vestuário adequado, dentre outros.

Em 58,1\% das comunidades eram oferecidas atividades aos familiares, que ocorriam majoritariamente de forma mensal. Além disso, $86 \%$ desenvolviam atividades conjuntas entre residente e família, na maioria das vezes, também de forma mensal.

Merece reflexão, contudo, o baixo percentual $(39,4 \%)$ de atendimento psicológico a essas famílias, também realizado predominantemente de forma mensal, além das visitas domiciliares, que aconteciam de uma forma esporádica (41,9\%).

Sobre o momento da alta terapêutica $90,7 \%$ das comunidades apresentava algum mecanismo de preparo do residente. Esses mecanismos envolviam acompanhamento e aconselhamento por equipe multiprofissional, visita à família, ressocialização e a realização de uma nova triagem.

\section{Discussão}

O presente estudo encontrou majoritariamente comunidades terapêuticas com residentes com perfil predominantemente caracterizado por: homem, adulto, usuário de drogas (Lícitas e ilícitas) e com renda familiar mensal inferior a um salário mínimo. Estudo sobre comunidades terapêuticas de Florianópolis, mostrou um perfil semelhante: homens adultos, com renda inferior a dois salários mínimos, desempregados e em uso das seguintes substâncias (Em ordem decrescente): álcool, cocaína, maconha, crack, cocaína injetável e tabaco(14).

Em relação às atividades de preparação do residente para a sua reinserção social, foi encontrado um baixo número de comunidades com essa disponibilidade, como oficinas profissionalizantes, cursos de informática e apoio educacional. A totalidade das comunidades relatou obrigatoriamente a prática de laborterapia, que é destacada como importante instrumento de reabilitação, reconstrução de cidadania e autonomia do sujeito, podendo ainda ser considerada o ponto central do programa terapêutico de qualquer comunidade. Porém, o ideal seria que a esta atividade fosse adicionada outras práticas e projetos para preparar o residente para a sua reinserção social ${ }^{(15)}$. Em contrapartida, defendese também a superação das práticas laborterápicas com vistas à construção de novas formas de sociabilidades, como, por exemplo, a construção de projetos de trabalho potencializadores de capacidades e de autonomia(16).

Resultados semelhantes foram encontrados em uma pesquisa que envolveu adolescentes residentes de uma comunidade terapêutica de ordem religiosa, na qual o programa terapêutico da comunidade envolvia quatro aspectos: a laborterapia, a convivência comunitária, a visita periódica a espaços culturais da cidade e o desenvolvimento de atividades profissionalizantes. Este último elemento era representado por aulas de computação, oficinas de violão, crochê e bordado. Estas atividades, entretanto, se encaixam mais como lúdico e terapêuticas do que profissionalizantes. Desta forma, essa comunidade apresentava dificuldades na preparação do residente para a sua reinserção social. Assunto recorrente na maioria das comunidades do presente estudo(5).

Conforme descrito anteriormente, o encaminhamento do residente ao mercado de trabalho é um grande desafio para a maioria das comunidades, visto que ainda é realizado de forma esporádica e não sistematizada. Na reinserção social esse encaminhamento é visto como essencial, uma vez que, o dependente químico enfrenta diversas dificuldades em sua reintegração à sociedade, entre elas a exclusão social que pode acarretar desemprego(17). Segundo uma pesquisa com 12 ex-residentes de comunidade terapêutica de Londrina (PR), 50\% dos egressos conseguiram colocação profissional logo após o desligamento institucional e $25 \%$ dos entrevistados encontravam-se desempregados ${ }^{(18)}$. Níveis de desemprego ainda mais elevados foram encontrados em uma pesquisa com usuários de crack atendidos em unidade de desintoxicação de um hospital de Porto Alegre (RS), em que $80 \%$ estavam desempregados ou autônomos ${ }^{(19)}$. Melhores resultados foram apresentados por uma pesquisa realizada em Portugal, com a participação de 150 ex-residentes de uma comunidade terapêutica local, em que foi constatado baixa taxa de desemprego $(20 \%)^{(20)}$.

Sobre o acompanhamento do dependente após a alta terapêutica, os resultados do presente estudo mostraram um pequeno número de comunidades terapêuticas que tentam fazer esse registro e acompanhamento dos egressos no mercado de trabalho, ação que poderia impactar de forma positiva às possíveis recaídas.

A participação familiar na reinserção social do dependente mostra-se bem estabelecida nas comunidades terapêuticas, com atividades conjuntas residente e família e visitas frequentes, na maior parte das comunidades. A importância da família na 
reinserção social do indivíduo é destacada também pela relevância para a segurança emocional e social do dependente durante essa fase (10). A família também é importante na reabilitação psicossocial que é obtida por meio de apoio, confiança e diálogo(21). Apesar disso, o número de comunidades que realiza atendimento psicológico à família do residente ainda é escasso. Esses resultados se comparam aos encontrados em uma pesquisa na qual os dependentes químicos destacaram a importância de sua família no processo de reinserção social, aliada a intervenção psicológica e atividades de prevenção à recaída. Em conformidade com os próprios dependentes desse estudo, as recaídas se manifestam na maioria das vezes por meio da fragilização e dos conflitos familiares, tema importante e que também merece reflexão ${ }^{(22)}$.

As visitas familiares dentro da comunidade terapêutica, seja de forma semanal ou mensal, constituem importante elo objetivando uma reabilitação plena. O retorno à família constituiu o primeiro passo para a reinserção social, sendo este mais fácil ou difícil dependendo do interesse e preocupação das mesmas $^{(18)}$

\section{Conclusão}

As ações e atividades voltadas para a reinserção social utilizadas pelas comunidades terapêuticas investigadas envolvem desde laborterapia até oficinas profissionalizantes, aulas de informática e apoio educacional. Porém, constata-se que estas atividades são limitadas a um pequeno número de comunidades.

Uma estratégia essencial para a reinserção social do dependente é a contínua interação de sua família com a comunidade terapêutica. Esse estudo demonstrou que esse convívio ocorre por meio de visitas e atividades conjuntas entre residente e família. Contudo, o atendimento psicológico às famílias e o tratamento conjunto (família e residente) ainda são pouco empregados por essas instituições.

Fundamental também é a inserção no mercado de trabalho após a alta terapêutica. Esse aspecto do processo de reinserção social ainda esbarra na falta de estruturação dessas comunidades. Existe ainda desconfiança e preconceito por parte de quem emprega um ex-residente, além da falta de qualificação profissional destes indivíduos em abstinência.

O papel desempenhado pelas comunidades na reabilitação psicossocial e reinserção do residente no convívio social é relevante. Existe ainda uma grande ausência de políticas públicas que envolvam a reintegração desses indivíduos e a superação da discriminação social. Essa é, possivelmente, a parte mais complexa do tratamento e o fracasso da reabilitação psicossocial que muitas vezes associa o indivíduo à dependência.

\section{Referências}

1. United Nations Office on Drugs and Crime (UNODC). Relatório Mundial sobre drogas 2012. [Acesso 12 out 2014]. Disponível em: http://www.unodc.org/ southerncone/pt/drogas/rela torio-mundial-sobre-drogas. html.

2. United Nations Office on Drugs and Crime (UNODC). Relatório mundial sobre drogas 2015. [Acesso 23 jun 2015]. Disponível em: http://www.unodc.org/documents/ wdr2015/World_Drug_Report_2015.pdf

3. Portaria n. 3088 (BR), de 23 de dezembro de 2011. Institui a Rede de Atenção Psicossocial para pessoas com sofrimento ou transtorno mental e com necessidades decorrentes do uso de crack, álcool e outras drogas, no âmbito do Sistema Único de Saúde. Brasília; 2011.

4. De Leon G. A comunidade terapêutica: teoria, modelo e método. São Paulo: Loyola; 2003.

5. Raupp LM, Milnitisky-Sapiro C. A "reeducação" de adolescentes em uma comunidade terapêutica: o tratamento da drogadição em uma instituição religiosa. Psicol Teor Pesq. 2008;24(3):361-8.

6. Silva MAR. Comunidade terapêutica: na mão ou na contramão das reformas sanitária e psiquiátrica? [Dissertação]. Florianópolis (SC): Universidade Federal de Santa Catarina; 2013. 265 p.

7. Silva GG, Pinto MR, Machineski GG. Percepção dos familiares de usuários de substâncias psicoativas em relação ao tratamento em comunidade terapêutica. Cogitare Enferm. 2013;18(3):475-81.

8. Duailibi LB, Severino R, Barbosa VMM, Ribeiro M. Reabilitação psicossocial e gerenciamento de caso. In: Ribeiro M, Laranjeira R., organizadores. O tratamento do usuário de crack. Porto Alegre: Artmed; 2012. p. 434-46.

9. Bonadio AN. Reabilitação psicossocial. In: Diehl A, Cordeiro DC, Laranjeira R, organizadores. Dependência química. Porto Alegre: Artmed; 2011. p. 267-73.

10. Pereira EL. Processo de reinserção social dos exusuários de substâncias ilícitas. 2009. [Acesso 13 mar 2014]. Disponível em: http://www.mpce.mp.br/esmp/publicações/ edi0012012/artigos/18Elain e.Lucio.Pereira.pdf

11. Pinho $\mathrm{PH}$, Oliveira MAF, Vargas $\mathrm{D}$, Almeida MM, Machado AL, Silva ALA, et al. Reabilitação psicossocial dos usuários de álcool e outras drogas: a concepção de profissionais de saúde. Rev Esc Enferm. 2009;43(2):1261-6. 
12. Lei Complementar n. 78 de 25 de março de 2010 (GO-BR). Altera a Lei Complementar n. 27, de 30 de dezembro de 1999, que cria a Região Metropolitana de Goiânia, autoriza o Poder Executivo a instituir o Conselho de Desenvolvimento da Região Metropolitana de Goiânia, a Secretaria Executiva e a constituir o Fundo de Desenvolvimento Metropolitano de Goiânia. Disponível em: http://www.gabinetecivil.go.gov.br/pagina_ leis. php?id=9457. [Acesso 1 mar 2014].

13. Morselli VL, Batista SHR, Rocha S. Cadastro Estadual das Comunidades Terapêuticas e Instituições Afins - Goiás. Eixo das Comunidades Terapêuticas / Fórum Goiano de Enfrentamento ao Crack e outras drogas; 2012.

14. Schneider DR, Sporh B, Leitão C, Accorsi M, Sacatamburlo N. Caracterização dos Serviços de Atenção à Dependência Química da Região da Grande Florianópolis. Relatório de Pesquisa. Universidade Federal de Santa Catarina; 2010. [Acesso 10 jul 2015]. Disponível em: http://psiclin.ufsc.br/files/2010/05/pesqui sa-1.pdf.

15. Fonseca JCF. Trabalho e dependência química: redes sociais e novas possibilidades de atenção à saúde. [Acesso 9 nov 2014]. Disponível em: http:// www.abrapso.org.br/si teprincipal/images/Anais_ XVENABRAPSO/601.\%20trabal ho\% 20 e $\% 20$ depend\%CAncia\%20qu\%CDmica.pdf.

16. Kinker FS. Enfrentamentos e construção de projetos de trabalho para a superação da laborterapia. Cad Ter Ocup. 2014;22(1):49-61.

17. Ganev E, Lima WL. Reinserção social: processo que implica continuidade e cooperação. Serv Soc Saúde. 2011;10(11):113-29.

18. Costa SF. O processo de reinserção social do dependente químico após completar o ciclo de tratamento em uma comunidade terapêutica. Serv Soc Rev. 2011;3(2):215-42.

19. Guimarães CF, Santos DVV, Freitas RC, Araújo RB. Perfil do usuário de crack e fatores relacionados à criminalidade em unidade de internação para desintoxicação no Hospital Psiquiátrico São Pedro de Porto Alegre (RS). Rev Psiquiatr. 2008;30(2):101-8.

20. Vieira CA. Comunidade terapêutica: da integração à reinserção. Toxicodependências. 2007;13(3):15-22.

21. Schonrr A, Hess ARB. Perspectivas do usuário de crack ao término do tratamento em comunidades terapêuticas quanto a sua reinserção social. /Trabalho de Conclusão do Curso de Psicologia da FACCAT; 2012/. 22. Scurssel R, Vasconcellos SJL. Dependência química: causas de recaídas na percepção do dependente químico. /Trabalho de Conclusão do Curso de Psicologia da FACCAT; 2010/. 